\title{
Usability of Passive Models for Energy Minimization of Transcutaneous Electrical Stimulation Possibilities and Shortcomings of Analytical Solutions of Passive Models and Possible Improvements
}

\author{
Aljoscha Reinert, Jan C. Loitz, Nils Remer, Dietmar Schroeder and Wolfgang H. Krautschneider \\ Institute of Nano- and Medical Electronics, Hamburg University of Technology, Eissendorfer Str. 38, Hamburg, Germany
}

Keywords: Transcutaneous Electrical Stimulation, Passive Model, Active Model, Energy, Minimization.

Abstract: $\quad$ Transcutaneous electrical stimulation is a more and more used rehabilitation technique for patients suffering from spinal cord injury or stroke. The commonly used pulse shape is the biphasic rectangular pulse, which leads to the question whether another, more efficient pulse shape exists that consumes less energy. In this study a passive model for electrical stimulation was develepod and an analytical analysis was performed. The resulting energy optimal pulse shape was then compared to the results of an active model. To improve the accuracy of the passive model, a simple ionic current correction was introduced, which leads to comparable results of an active model. Concluding it can be said that passive models are a good approach to give notions of some effects, but have to be extended to fit reality.

\section{INTRODUCTION}

Transcutaneous electrical stimulation (TES) is known to be used as a rehabilitation technique for patients suffering from spinal cord injury or stroke since the 1970s (Knutson et al., 2007). The most commonly used pulse shape for electrical stimulation is the biphasic rectangular pulse with a short interphase with the attributes stimulation amplitude $(m A)$, pulse duration $(\mu s)$ and stimulation frequency $(\mathrm{Hz})$ (Hunter Peckham, 1999). The relation between amplitude and duration for energy optimized stimulation with rectangular pulses is shown in the strength duration curve for biphasic rectangular pulses. As modern electronics allow not only to change amplitude, duration and frequency, but also the shape of the stimulation pulse, the question occurred whether it is possible to find an optimized pulse shape that consumes the least energy.

This question was discussed several times in the past (Jezernik and Morari, 2005; Wongsarnpigoon and Grill, 2010; Krouchev et al., 2014). Most of these papers do not differentiate between the energy that has to be provided by the battery of the stimulator and the energy that is applied to the patient. This paper focuses on the energy transmitted to the patient, as the possible harm for the body should be minimized. It should also be noted that the optimal pulse shape is one single shape, with a distinct amplitude, as boundary conditions like maximum amplitude or fixed pulse duration contradict the definition of energy optimized.

The goal of this study is to develop a passive model, i.e. based only on capacitors and resistors, to find an analytical solution and to figure out how this solution can be used for the prediction of action potentials. In the second step this passive model is compared to an active model, which uses differential equations to model the ionic current in the axon. In the third step we investigate how the passive model can be improved to match the active model without dramatic increment of complexity.

\section{PASSIVE MODEL}

\subsection{Development of a Passive Model}

For transcutaneous electrical stimulation a lot of different models with varying complexity are available (Kuhn and Keller, 2005; Kuhn et al., 2009; Villarreal et al., 2013). Those equivalent circuits are often built as passive models, only consisting of capacitors and resistors, as electrical parameters are known and simulating passive models is very fast and accurate. In the following, the development of an equivalent cir- 
cuit for the prediction of triggering an action potential is described.

The simplest equivalent circuit model of the axon is shown in figure 1. It consists solemnly of one RCcircuit, which is the merged form of two serial RCcircuits (one for entering the axon, one for leaving the axon) with the same electrical properties. The axon membrane is represented by the capacitor as all leakage currents and surrounding tissue is merged into the parallel resistor. In this model the current path from the electrodes to the axon is neglected. It can easily be seen that this is a simplification that dramatically changes the reliability of the results. Therefore this model has to be extended.

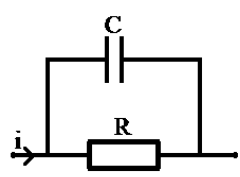

Figure 1: Most simple passive model of an axon, consisting only of a membrane capacitor and resistor.

To include the losses in the current path between electrodes and axon, another series resistor $\left(R_{2}\right)$ has been added. The corresponding equivalent circuit is shown in figure 2 . The resistor $R_{2}$ merges the resistive losses of the current path to and from the axon, as the two serial resistors for both paths can be merged into one resistor. The same is true for all following models and their serial impedance. The added resistor dramatically changes the behavior of the model, but it is still not quite accurate. In this model there is still the assumption that all of the stimulation current is flowing through the axon and the surrounding tissue, neglecting a possible current path, which is close to the electrodes, far away from the axon. This is also a simplification as all losses in the electrode skin interface is neglected, which can be extremely high because of the high electrode size when performing transcutaneous electrical stimulation.

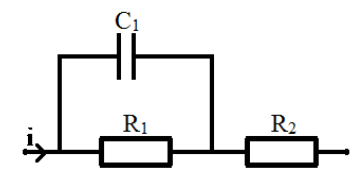

Figure 2: Passive model that considers losses in cables and tissue.

To consider this interface and the capacitive behavior of the tissue another series RC circuit $\left(R_{2} C_{2}\right)$ has been added, as shown in figure 3 .

The amount of current that flows into the tissue and passes the tissue around the axon can be considered by adding an additional RC circuit in parallel to

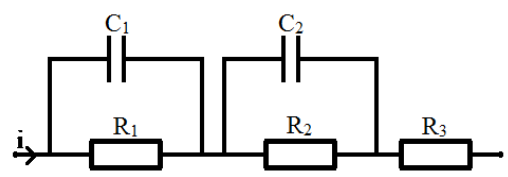

Figure 3: Passive model that considers the electrode-skininterface and capacitive behavior of tissue.

the existing RC-circuits $\left(R_{3} C_{3}\right)$. The amount of current that does not enter the path required for stimulation is determined by the impedance ratio of the two parallel paths, shown in figure 4 .

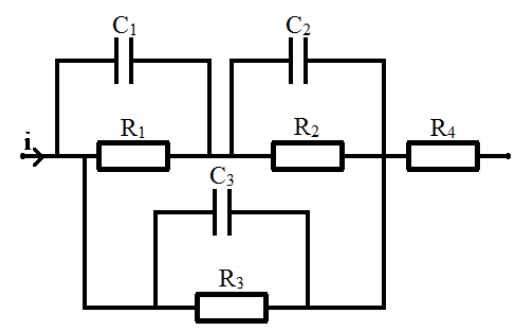

Figure 4: Passive model that considers the capacitive behavior of tissue and the distribution between current that flows through the axon and current that does not.

As the electrode skin interface and tissue behavior can no longer be merged together into one RC-circuit, the last step is to add another series RC circuit to the existing schematic to model the electrode skin interface and tissue path to the axon $\left(R_{4} C_{4}\right)$, see figure 5 .

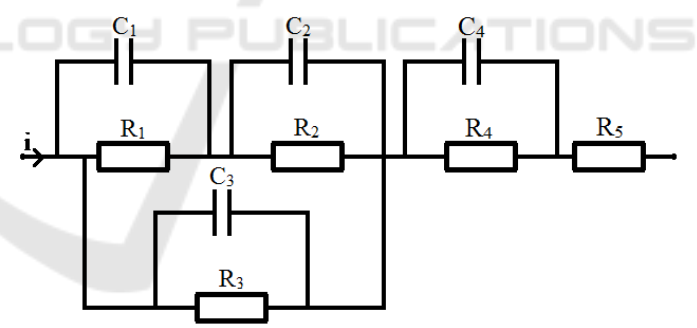

Figure 5: Passive model that considers the electrode-skininterface,capacitive behavior of tissue and current distribution.

This model can now be extended with additional RC-circuits that represent single layers of skin, fat or muscle, forming an RC-network. For a given frequency this network can always be simplified to the last step of the model given in figure 5. All these models focus on predicting action potentials of a single node of Ranvier in the axon, therefore ignoring contribution of neighboring cells that can be modeled with the activating function (Rattay, 1999). Also only the membrane potential is calculated as this is necessary to decide whether or not an action potential is triggered. The prediction stops at the moment when an action potential is triggered, as the differential equa- 
tions needed to describe an action potential (Hodgkin and Huxley, 1952; McIntyre et al., 2002) and their resulting ionic currents are not included in passive models.

\subsection{Analytical Solutions for Energy Minimization}

When finding the optimal pulse shape, it is necessary to minimize the energy for a fixed change of the membrane potential $V_{m}$ or to maximize the change of the potential $V_{m}$ with a fixed amount of energy $E$. The third option would be maximizing the efficiency, therefore maximizing $\frac{V_{m}}{E}$. This includes minimizing the energy and maximizing the membrane potential at the same time. In this paper the focus lies on the energy that is dissipated in the tissue, not the energy that is provided by the battery of the stimulator. As for most stimulation devices a constant voltage source is used, which gives for the latter

$$
E=\int V_{\text {source }} \cdot i d t=V_{\text {source }} \cdot Q
$$

as $V_{\text {source }}$ is constant. Optimizing this means optimizing for charge, as the energy is only determined by the integral of the current.

Calculating the energy in the tissue is given by a varying voltage and current. Choosing the first model, shown in figure 1, gives us the following equations. The solution is straight-forward. As the voltage drop over the membrane is determined by

$$
i_{C}=C \cdot \frac{d V_{m}}{d t}
$$

and the energy loss over the resistor is proportional to the time

$$
E_{R}=\int V_{m} \cdot i_{R} d t
$$

an infinitely high and infinitely short current spike is the optimal solution. This would lead to an instantaneous voltage spike over the membrane capacitance and therefore immediately trigger an action potential without any losses over the resistor $R$.

Adding the series resistor in the second model invalidates this solution, as the power loss in the series resistor can be calculated by

$$
E_{R 2}=\int i^{2} \cdot R_{2} d t
$$

thereby limiting the maximum amplitude of the current, as energy scales with the square of the current. The efficiency is defined as:

$$
\frac{d V_{m}}{d E}=\frac{d V_{m}}{d \int\left(V_{m} \cdot i+i^{2} \cdot R_{2}\right) d t}
$$

Differentiating this equation and setting it equal to zero will give us the maximums and minimums and therefore the point of optimal energy efficient stimulation. Doing this will lead to a complex differential equation, where solving with Laplace leads to a convolution of the current $i$ with an exponential function $e^{-\frac{t}{\tau}}$, which is very complex to solve analytically, as the current itself is unknown. The solution however is reasonable, as a convolution with an exponential function is nothing else than the charge stored in the capacitor, depending on the shape of the pulse and the time elapsed.

To avoid this problem a minimization approach for constant currents and infinitesimal time interval has been chosen. The system is at every instant defined by the charges stored in the capacitors and their corresponding voltages. Applying an external constant current $I_{1}$ to the system for a duration of $d t$ will lead to a change of the system state. At the point $t+d t$ the system a new constant current $I_{2}$ can be applied to the system. For each single point in time the efficiency can be calculated by using the current state of the system. Always choosing the current $I$ with the highest efficiency for every single point then leads to the energy optimized pulse shape $i$.

Following this logic claims that only one optimal pulse shape exists. This is true as long as no additional boundary conditions are applied. In literature the maximum amplitude or maximum pulse duration is often applied as an additional boundary condition, which can lead to different, less efficient results. Setting the pulse duration to a predefined value is critical only if this value is too small, changing the appearance of the shape. A longer pulse duration only leads to a shift of the optimized shape (Sahin and Tie, 2007).

As stated above, the first step is to find the most efficient waveform, which is given by maximizing $\frac{d V_{m}}{d E}$. The voltage drop along the $R_{1} C_{1}$ circuit in figure 2 can be calculated as:

$$
V_{1}=\left(V_{0}-R_{1} I\right) \cdot e^{\frac{-t}{R_{1} C_{1}}}+R_{1} I
$$

$V_{0}$ defines the voltage drop at the capacitor at $t=0$. The voltage drop over the resistor $R_{2}$ is defined as

$$
V_{2}=R_{2} I
$$

The assumption that for each given voltage an optimal current exists is only valid for an infinite short amount of time $d t$, which leads to the optimization problem of maximizing the efficiency

$$
X=\frac{\frac{d V}{d t}}{\frac{d E}{d t}}=\frac{\frac{d V}{d t}}{P}
$$

The power can be calculated by the product of voltage and current. Minimization of this equation with 
respect to I leads to

$$
I\left(V_{1}\right)=\frac{V_{1}}{R_{1}}+V_{1} \sqrt{\frac{R_{1}+R_{2}}{R_{1}^{2} R_{2}}}
$$

Combining this equation with the original equation for the voltage drop then gives us a solution with the shape of $i=k \cdot e^{\frac{t}{\tau}}$. The result of an exponential function as an optimal pulse shape for passive models has also been derived by (Wongsarnpigoon et al., 2010). Adding additional RC-circuits as shown in in figure 5, does not change the linearity of the differential equations, always leading to a result in the shape of

$$
i=\Sigma k_{n} \cdot e^{\frac{t}{\tau_{n}}}, k_{n} \geq 0
$$

Therefore passive models will always lead to exponentially shaped pulse shapes as an optimal result, no matter how many and detailed RC circuits are added.

However, experiments like (Wongsarnpigoon et al., 2010) already showed that the exponential increase is not the optimum pulse shape in reality. We can conclude that there is still something important missing, making passive models as they are right now insufficient for the prediction of action potentials.

\section{ACTIVE MODEL}

\subsection{Active Model Simulation Results}

To build models that are closer to reality, the ionic currents and active behavior of axons have to be modeled as well. Active models that use the differential equations proposed by (McIntyre et al., 2002) are used to determine this behavior. As a basis for calculation a 3D finite element (FE) model was used to model the forearm and calculate the extracellular potentials (Loitz et al., 2015) and is shown in figure 6. To minimize calculation time, the system response to

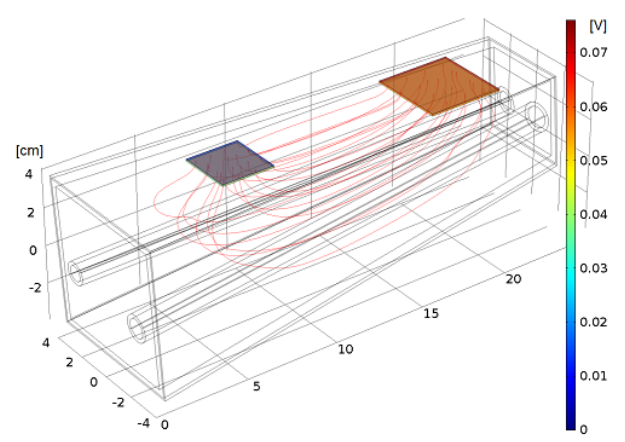

Figure 6: Simplified finite element COMSOL model of a human forearm. a rectangular pulse of $1 \mathrm{~mA}$ and $1 \mu \mathrm{s}$ was simulated in COMSOL and post-processed in Matlab. This pulse was then extended to $80 \mu$ s and the amplitude was varied until the minimum amplitude to trigger an action potential was found. This amplitude was set as the maximum for the following steps. The duration of $80 \mu s$ was extended to $160 \mu s$ and split into multiple segments as shown in figure 7. To keep calculation time low, a number of four segments was chosen. For each of this rectangular segments the amplitude was varied from minimum to maximum in steps of $10 \%$ of the maximum amplitude until an action potential was triggered. All the valid results are then compared and the solution with the least amount of consumed energy was saved. This can be used as a first approach to determine the optimal pulse shape. Using only the electrical parameters from COMSOL

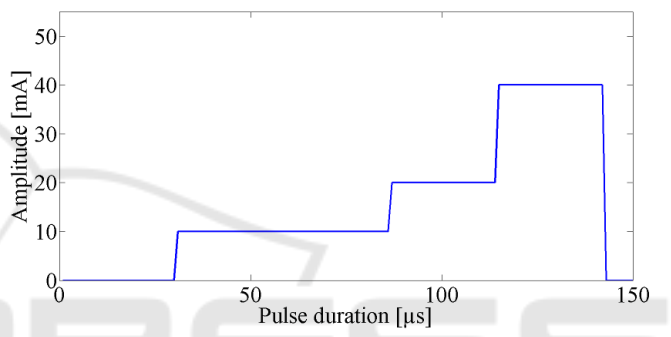

Figure 7: Finite Element Simulation results with Passive Model and split pulse.

and applying the results to Matlab gives a roughly exponential shape as a result for an optimal pulse shape, supporting the analytical solution found with the passive model as shown in figure 7. Now adding the Hodgkin-Huxley equations to this model leads to an interesting effect of decreasing current for the last of the four rectangular pulses, see figure 8 . This can be explained with the ionic current that starts to flow before threshold is reached, therefore contributing to the total current needed for stimulation. As the ionic current can be calculated with the Hodgkin-Huxley equations and is approximately exponential at the beginning it decreases the amount of external current that is needed. This effect can be made more clear by increasing the amount of segments from four to a higher value, smoothing the shape of the stimulation pulse. On the other hand this dramatically increases the simulation time, as all combinations of amplitudes have to be computed and compared. (Meza-Cuevas et al., 2012) experimentally showed that a pulse shape with decreasing amplitude like sinusoidal is suited best to trigger an action potential. 


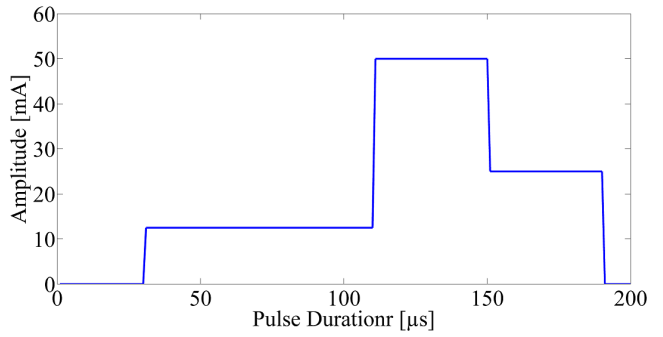

Figure 8: Finite Element Simulation results with Active Model.

\subsection{Semi-Active Model}

To improve the passive models in a way that they can be used to accurately predict action potentials an extension is needed. As this extension can no longer be passive, the proposal is to use semi-active models. These models are based on passive models, but have a single, very limited active component, that can be computed after the passive solution. Looking at the Hodgkin-Huxley equations, it is possible to approximate the behavior of the ionic current at the beginning of the stimulation with an exponential increasing function. After reaching the threshold potential this approximation is no longer valid as the additional ionic currents now dominate. The proposal is to include the ionic current as a fixed, exponentially increasing current, that has to be subtracted from the stimulation current, leading to a shape that can be approximately written as:

$$
i=k_{\text {stim }} \cdot e^{\frac{t}{\tau_{\text {stim }}}}-k_{\text {ion }} \cdot e^{\frac{t}{\tau_{\text {ion }}}}
$$

The given equation consists of the stimulation current and the ionic current that can be scaled to match the optimization problem and gives fast results that are comparable to those of an active model as shown in figure 9. The passive stimulation current is given by

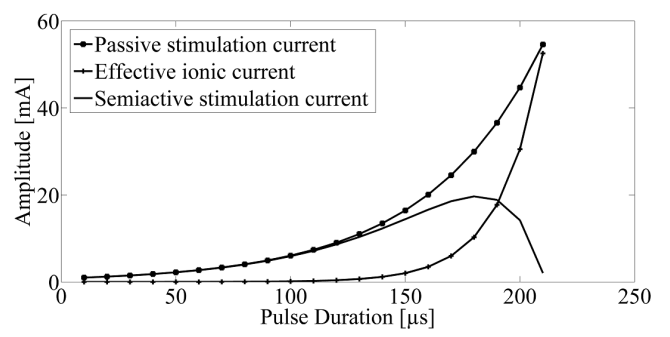

Figure 9: Exponential increase pulse with ionic current and their difference.

the result of the passive model. The effective ionic current is the representative current that has to be subtracted from the external stimulation current to cause the same effects as the internal ionic current.
As only a small amount of stimulation current flows through the axon the magnitudes of the real ionic current and the effective ionic current differ a lot. To determine the parameters $k_{i}$ on and $\tau_{i}$ on two fixed points are needed. The first fixed point is at the end of the stimulation, as the active ionic current is now high enough to carry the action potential all by itself, being equal to the equivalent passive model external stimulation current. This results in a semiactive stimulation current of zero at the end of the pulse. The second point determines the maximum of the semiactive pulse shape and is in the range of $50 \%$ to $80 \%$ of the pulse duration, depending on the model. Determination of the exact position has to be investigated further, a value of $66 \%$ showed very good results and is recommended.

\section{CONCLUSIONS}

In this study a passive model with stepwise increasing complexity has been developed that enables prediction of action potentials with given pulse shapes and allows to analytically calculate the optimal pulse shape for energy minimization in the tissue. The results of these passive models are always exponentially increasing shaped pulses. Comparing these results with experiments leads to the problem that even though passive models already lack the ability to model the behavior during an action potential, they also fail to accurately model the behavior shortly before an action potential is triggered. To improve the passive model, a scalable ionic current has been introduced that represents the behavior of the ion channels. With these both combined results that are comparable to these of an active model can be achieved. Passive models are therefore a good way to quickly get a notion of some effects, as this approach shows that the results of an active and passive model may differ, but never in a completely different way.

\section{ACKNOWLEDGEMENTS}

This work was supported by a grant from the Federal Ministry of Education and Research (BMBF, ESiMED [16M3201]).

\section{REFERENCES}

Hodgkin, A. L. and Huxley, A. F. (1952). A quantitative description of membrane current and its application to 
conduction and excitation in nerve. The Journal of physiology, 117(4):500-544.

Hunter Peckham, P. (1999). Principles of electrical stimulation. Topics in spinal cord injury rehabilitation, $5(1): 1-5$.

Jezernik, S. and Morari, M. (2005). Energy-optimal electrical excitation of nerve fibers. Biomedical Engineering, IEEE Transactions on, 52(4):740-743.

Knutson, J. S., Harley, M. Y., Hisel, T. Z., and Chae, J. (2007). Improving hand function in stroke survivors: a pilot study of contralaterally controlled functional electric stimulation in chronic hemiplegia. Archives of physical medicine and rehabilitation, 88(4):513-520.

Krouchev, N. I., Danner, S. M., Vinet, A., Rattay, F., and Sawan, M. (2014). Energy-optimal electricalstimulation pulses shaped by the least-action principle. PloS one, 9(3).

Kuhn, A. and Keller, T. (2005). A 3d transient model for transcutaneous functional electrical stimulation. In International functional electrical stimulation society conference, volume 10, pages 385-387.

Kuhn, A., Keller, T., Lawrence, M., and Morari, M. (2009). A model for transcutaneous current stimulation: simulations and experiments. Medical \& biological engineering \& computing, 47(3):279-289.

Loitz, J. C., Reinert, A., Schroeder, D., and Krautschneider, W. H. (2015). Impact of electrode geometry on force generation during functional electrical stimulation. Current Directions in Biomedical Engineering, 1(1):458-461.

McIntyre, C. C., Richardson, A. G., and Grill, W. M. (2002). Modeling the excitability of mammalian nerve fibers: influence of afterpotentials on the recovery cycle. Journal of neurophysiology, 87(2):995-1006.

Meza-Cuevas, M., Schroeder, D., Krautschneider, W. H., et al. (2012). Neuromuscular electrical stimulation using different waveforms: Properties comparison by applying single pulses. In Biomedical Engineering and Informatics (BMEI), 2012 5th International Conference on, pages 840-845. IEEE.

Rattay, F. (1999). The basic mechanism for the electrical stimulation of the nervous system. Neuroscience, 89(2):335-346.

Sahin, M. and Tie, Y. (2007). Non-rectangular waveforms for neural stimulation with practical electrodes. Journal of neural engineering, 4(3):227.

Villarreal, D. L., Schroeder, D., , and Krautschneider, W. H. (2013). Equivalent Circuit Model to Simulate Neurostimulation by using Different Waveforms. In ICT Open 2013.

Wongsarnpigoon, A. and Grill, W. M. (2010). Energyefficient waveform shapes for neural stimulation revealed with a genetic algorithm. Journal of neural engineering, 7(4):046009.

Wongsarnpigoon, A., Woock, J. P., and Grill, W. M. (2010). Efficiency analysis of waveform shape for electrical excitation of nerve fibers. Neural Systems and Rehabilitation Engineering, IEEE Transactions on, 18(3):319-328. 